\title{
EL SÍNDROME DE DANDY WALKER Y SU INTERVENCIÓN EN LA INFANCIA
}

\author{
Dandy-Walker syndrome and treatment in children \\ Isabel M. a García Caballero (isamgarcia@correo.ugr.es). Universidad de Granada \\ Fecha de recepción: 28 de mayo de 2012 \\ Fecha de aceptación: 11 de junio de 2012 \\ Localizador: http:www.ugr.es/local/miguelgr/ReiDoCrea-Vol.1-Art.7-Garcia.pdf
}

\section{RESUMEN}

El Síndrome de Dandy Walker es una anomalía congénita del cerebelo y del IV ventrículo, que aparece normalmente en la infancia y recibe múltiples nominaciones. Su característica más común es la hidrocefalia y en la mayoría de los casos, las causas se desconocen. El artículo se centra en gran parte en el análisis de un caso práctico de un niño de cinco años, con dificultades en la movilidad debido al retraso psicomotor y sin habla, cuyo tratamiento está basado en el control de los movimientos, en la estimulación del balbuceo y en el aprendizaje de un sistema alternativo de comunicación.

Palabras clave: Síndrome de Dandy Walker*, Infancia, lengua, necesidades educacionales y técnicas de comunicación.

\begin{abstract}
Dandy-Walker Syndrome is a congenital anomaly of the cerebellum and the forth ventricle, which usually appears in childhood and has many names. Its most common characteristic is hydrocephalus and in most cases, the causes are unknown. This article is mainly focused on the analysis of a practical case of a five-year-old child, with mobility difficulties due to psychomotor retardation and without speech, whose treatment is based on the control of movements, on the stimulation of babbling and on the acquisition of an alternative communication system.
\end{abstract}

Key words: Dandy-Walker Syndrome*, childhood, language, educational needs and communication techniques.

*No está incluido en Thesauro de la UNESCO, pero consideramos que es un concepto importante, ya que sobre él se centra el artículo, y por lo tanto debe ser incluido. 


\section{INTRODUCCIÓN}

El Síndrome de Dandy Walker (SDW) es una anomalía congénita del cerebelo y del cuarto ventrículo. Recibe otras nominaciones, como malformación de Dandy Walker, quiste, deformidad, malformación de Luschka y Magendie, etc. muchos de estos nombres han sido dados tras descubrimientos patológicos. Para Goyenechea Gutiérrez y Tablada, "lo más apropiado es denominar la patología como Síndrome de Dandy Walker, pues se trata de un conjunto de síntomas y signos secundarios a una disembriogenesis del cerebro medio y por tanto las alteraciones anatómicas que aparecen depende de la severidad del trastorno malformativo" (Goyenechea Gutiérrez y Hodelín Tablada).

La etiología es muy heterogénea. Es más frecuente en el sexo femenino, con una relación de $3: 1$ y su incidencia se estima entre 1 cada 25.000 y 1 cada 30.000 , engloba el $10 \%$ de todos los casos de hidrocefalia (Rodríguez y Cabal, 2010).

La característica más relevante es la hidrocefalia, dilatación anormal de los ventrículos del encéfalo por acumulación de líquido cefalorraquídeo, en este caso provocada por la expansión quística del cuarto ventrículo. Otros autores (Greenberg, Incesu, Khosla y Klein) manifiestan que para que se produzca el SDW son necesarias tres características: tener el IV ventrículo quístico, una fosa posterior agrandada y la presencia de una aplasia o hipoplasia cerebelosa. Se ha llegado a considerar característica importante la presencia de atresia de los agujeros de Luschka y Magendie, pero lo cierto es que puede no estar presente.

En la mayoría de los casos, las causas se desconocen, pero puede descubrirse antes del nacimiento, por medio de ultrasonidos; o en el primer año de vida debido a la hidrocefalia.

Puede haber presencia de una protuberancia en la parte de atrás de la cabeza; o un "collar de pelo" encima de ésta y la piel puede ser rojiza por esta zona. También pueden aparecer problemas congénitos del corazón, malformaciones en la cara, los dedos, etc. y otras anomalías en el sistema nervioso central. Son frecuentes modelos anormales de respiración, retraso en el sistema motor, hipotonía, etc. sin olvidarse de los problemas de aprendizaje. Algunas personas pueden sufrir este síndrome toda su vida y no manifestarse, al igual que algunos niños pueden tenerlo asociado a otros síndromes dando lugar a complicaciones muy severas.

A continuación se muestran tres apartados aclaradores de algunas anomalías y síndromes que pueden ir asociados a esta malformación (Osorio, Rodríguez, Pizarro, Koller, Paredes y Zúñiga).

1. Anomalías congénitas asociadas al SNC relacionadas con el SDW (70\%):

- Disgenesia cuerpo calloso (20-25\%)

- Holoproscencefalia (25\%)

- Displasia del giro cingulado (25\%)

- Microcefalia

- Espina bífida

- Meningocele lumbosacro

2. Anomalías congénitas no asociadas al SNC relacionadas con el SDW (20-33\%):

- Malformaciones orofaciales y palatinas (6\%)

- Anomalías cardíacas

- Riñones poliquísticos 
- Disgenesia retinal

- Síndrome de Meckel-Gruber

3. Síndromes asociados al SDW:

- Síndrome de Aase-Smith

- Síndrome de Aicardi

- Síndrome Cerebro-oculomuscular

- Síndrome de Coffin-Siris

- Síndrome de Cornelia de Lange

\section{HISTORIA}

Según Pascual-Castroviejo, en 1887, Sultton fue el primero en describir un quiste de fosa posterior con hipoplasia del cerebelo; en 1914, Dandy y Blackfan 1914 describen lo que constituiría la tríada típica de la afección, posteriormente en 1942, Taggart y Walker, publicaron algunos casos y lo relacionan con la presencia de atresia de los agujeros de Luschka y Magendie; pero no fue hasta 1954 en que Benda propone denominar al cuadro SDW, comprobando que la entidad se debe a un desarrollo embriológico anormal del cerebro medio.

Aparecen después dos publicaciones de D’Agostino, en 1963 y Hart y colaboradores en 1972, donde se definen las características básicas. Hasta 1984 sólo se habían reportado 300 enfermos, de ellos 16 con una posible predisposición genética (Youmans JR.). Ese año Hirsch y colaboradores (Hirsch, Pierre-Kahin, Reinier, 1984) publicaron una revisión de 40 pacientes donde demostraron, las variaciones anatómicas existentes, destacándose las múltiples malformaciones de otros órganos y sistemas. Con las modernas técnicas imagenológicas, hoy son variadas las series que presentan amplias casuísticas, contribuyendo a un mejor estudio y seguimiento de los enfermos.

\section{CUADRO CLÍNICO}

Las manifestaciones clínicas dependen de: la severidad, las malformaciones asociadas, la edad y el momento del diagnóstico. Lo mejor sería que se llevara a cabo un diagnóstico prenatal mediante estudios ultrasonográficos a todas las madres en riesgo. La mayoría de los enfermos presentan síntomas durante la lactancia.

Una característica clínica importante es la presencia de una fosa posterior grande. La presencia de malformaciones en el tallo cerebral puede ocasionar dificultades de succión, deglución, respiración y cardiorespiratorias. También es frecuente la hipertensión endocranea con ataxia, espasticidad y dificultad en la motricidad fina. Suelen presentar también retraso mental.

"Es importante mencionar que se han detectado personas asintomáticas en la edad adulta que se han diagnosticado de manera incidental por motivos ajenos al síndrome de Dandy Walker" (Goyenechea y Hodelín Tablada).

\section{PRONÓSTICO}

El pronóstico varía según las anomalías asociadas. Si las malformaciones son múltiples y las anomalías muy severas pueden afectar gravemente a órganos y sistemas y ocasionar la muerte. Que se da aproximadamente en el $50 \%$ de los casos y si se asocia con anomalías severas asciende al 83\% (Incesu y Khosla). Por el contrario, el diagnóstico es favorable cuando se trata adecuadamente la hidrocefalia en los primeros años de vida con cirugía. 


\section{ANÁLISIS DE UN CASO}

Trabajamos con el caso de un niño de 5 años, N.J, nacido en el 2006. A los siete meses de embarazo ya se presentaban problemas y en los primeros días de vida se diagnosticó el síndrome. Permaneció unas semanas en la UCI como consecuencia de la asfixia que sufrió durante la cesárea. Su situación familiar es favorable.

N.J, además de presentar el SDW e hidrocefalia, tiene un 54\% de retraso madurativo, retraso del lenguaje, hipotonía muscular, dificultades motoras y no controla esfínteres.

Suele obsesionarse por un juguete y la conducta que presenta cuando algo no le gusta es la de dar manotazos. Tiene intención comunicativa, expresada a través de gestos, expresiones faciales, señalamientos, etc.

\section{OBJETIVOS}

Los objetivos que hemos trabajado con N.J se dividen en dos grupos:

\section{Psicomotrices}

- Adquirir la coordinación y el control dinámico de su cuerpo.

- Potenciar la motricidad gruesa.

- Proporcionar actividades de juego y movimiento.

- Favorecer la comunicación corporal.

\section{Lenguaje}

- Realizar actividades previas al lenguaje oral.

- Estimular el balbuceo.

- Imitar sonidos que percibe.

- Comprender mensajes sencillos.

- Aumentar los tiempos de atención sostenida.

Estas son metas planteadas para todo el ciclo, por lo que no todos están cubiertos por las actividades propuestas.

\section{METODOLOGÍA}

\section{Psicomotricidad}

Las sesiones están estructuradas, teniendo cada una de las cuales asignada unas actividades y objetivos. Las fases que se siguieron en las sesiones son: calentamiento, desarrollo o actividades programadas y relajación. Se parte siempre de lo que el niño sabe, para motivar al alumno a participar y realizar más movimientos.

\section{Lenguaje}

Sólo se impartía una sesión, de 45 minutos, a la semana. Las sesiones se dedicaban a la estimulación de los músculos fonoarticulatorios mediante terapia miofuncional, estimulación del balbuceo y aprendizaje de signos que sirvan como medio alternativo de comunicación.

\section{APLICACIÓN DE LAS INTERVENCIONES}

\section{Psicomotricidad}

Actividad 1: Con el niño boca abajo, llamamos su atención con objetos vistosos e ir levantando el objeto para que el niño levante la cabeza. 
El objetivo es que el niño controle su cabeza. La duración es de unos tres minutos, descansando si es necesario.

Actividad 2: Ponemos al niño sentado en una alfombra y le sujetamos con nuestras manos por la espalda, se le hará caer levemente hacia delante para que vuelva a la posición anterior.

El objetivo es que el niño se mantenga sentado. La duración es de unos tres minutos aproximadamente.

Actividad 3: Colocamos al niño en posición de gateo, manteniéndolo con nuestras manos y eliminando cada vez más nuestra ayuda. Inicialmente es suficiente que se apoye sobre los antebrazos para pasar a apoyarse sobre las manos.

El objetivo es que el niño comience a gatear, la duración es de unos cinco minutos.

Actividad 4: Con el niño en posición de gateo colocamos objetos atractivos a su alrededor para hacer que se mueva.

Al igual que el ejercicio anterior, el objetivo es gatear, la duración es de unos cinco minutos.

Actividad 5: Ponemos al niño al principio de unas escaleras con poca pendiente y estimulamos al niño para que la suba gateando.

El objetivo es que el niño gatee y sea capar de subir escaleras él solo o con nuestra ayuda. La duración es de unos tres minutos.

Actividad 6: Cogemos al niño de ambas manos y lo ponemos de pié. Intentamos que de varios pasos con nuestra ayuda.

El objetivo es estimular que el niño de pasos. La duración es de unos cinco minutos, pudiendo ampliar o acortar la actividad dependiendo del estado del niño.

Actividad 7: Puesto el profesor a un metro de distancia del niño, le anima con sus expresiones a que llegue hasta él sin ninguna ayuda.

El objetivo es, como el anterior, estimular que el niño de pasos. La duración podrá variar pero oscilará entre tres y seis minutos.

\section{LENGUAJE}

Durante los primeros 10 minutos se realiza Terapia miofuncional. Un masaje (pequeños círculos) en la cara, estimulando y relajando así los músculos fonoarticulatorios necesarios para la fonación, incidiendo sobre todo en la zona de las mejillas, cuya movilidad necesitamos para la producción del habla; e incidiendo también en los labios, para que estos consigan un tono superior.

Acompañamos los masajes con una canción infantil lenta y suave, para que sea más fácil relajar al niño, ya que suele estar muy nervioso y activo.

A continuación se procede a la emisión de las vocales y balbuceos. Le enseñamos al niño fichas con las vocales, con colores llamativos para captar su atención. Emitimos los sonidos de las vocales hasta que el niño los imite. Esta actividad puede llevarnos unos 10 minutos. Si el niño se pone nervioso volvemos a masajear las mejillas mientras le cantamos una canción. 
El resto del tiempo de las sesiones se dedica al aprendizaje de un sistema alternativo de comunicación, que en este caso, son los signos de la Lengua de Signos Española. Realizamos el signo mientras lo pronunciamos varias veces, hasta que el niño lo imite. Es bueno que se asocien los signos con los objetos reales. Los signos trabajados son: música, agua, oso, yogur, pelota, papá y mamá.

\section{RECURSOS}

Los recursos utilizados para el programa de intervención han sido los disponibles en el centro. No se ha necesitado materiales ajenos al centro.

\section{Personales}

- Maestro de apoyo, para los ejercicios de psicomotricidad.

- Maestro de Audición y Lenguaje.

\section{Materiales}

- Objetos llamativos y con colores vivos que pudieran llamar la atención del niño, como pelotas, llaves, sonajeros, etc.

- Una alfombra o colchoneta sobre la cual poder tumbar al niño.

- Unas escaleras con poca pendiente.

\section{CONCLUSIONES}

Como profesionales de la educación debemos conocer algunos de los síndromes raros, ya que éstos tienen repercusiones sobre el aprendizaje de los niños y niñas, ámbito sobre el que tenemos que trabajar. Existe un reducido número de materiales sobre estos síndromes, por lo que la búsqueda ha sido compleja y laboriosa. En el artículo se da a conocer el Síndrome Dandy Walker de una forma sencilla y cercana, que creemos que puede ser de utilidad para trabajos posteriores.

Pero más importante aún es la intervención, porque de nuestro trabajo va a depender el desarrollo de sus capacidades, la evolución del aprendizaje, el aumento de la autonomía del niño/a, etc. Para la aplicación de un correcto programa de intervención debemos tener conocimientos sobre el síndrome, las causas, el ámbito..., pero lo más importante es conocer las características, las consecuencias del síndrome, es decir, las repercusiones que tiene el síndrome en el aprendizaje del niño o niña y sobre eso, elaborar un programa de intervención que sea específico del niño/a con el/la que tenemos que tratar, ya que dentro de un mismo síndrome podemos encontrar diversidad de casos diferentes.

Las semanas de intervención con N.J han sido muy gratificantes. Ha sido un trabajo duro, ya que el comportamiento del niño no siempre era favorable y hemos tenido que interrumpir continuamente las sesiones. Los avances han sido perceptibles, aunque esperamos que al finalizar el ciclo N.J haya alcanzado todos los objetivos. En cuanto al tratamiento debemos decir que aunque las sesiones de psicomotricidad y de lenguaje son muy importantes, también lo es centrar la atención en la integración del niño. No podemos pretender que realice las actividades igual que sus compañeros y compañeras debido a sus limitaciones, pero si hemos conseguido en la mayoría de las ocasiones que comparta momentos con los demás, como realizar actividades por parejas o llevar a cabo las sesiones de psicomotricidad con varios niños/as. N.J es un niño feliz, que se esfuerza mucho para conseguir los objetivos propuestos y que siempre nos ha respondido positivamente ante los refuerzos dados, de él hemos 
aprendido que con esfuerzo e ilusión podemos dar pequeños pasos que nos lleven a grandes logros.

\section{REFERENCIAS BIBLIOGRÁFICAS}

Agostino, A.N., Kernoham, J.W. y Brown J.R. (1963). The Dandy Walker síndrome. Journal of Neuropathology and experimental neurology, 22, 450-470.

Goyenechea Gutiérrez, F. y Hodelín Tablada, R. Síndrome de Dandy Walker. Recuperado el 9 de mayo del 2012 de http://www.sld.cu/galerias/pdf/sitios/neuroc/dandy walker.pdf

Hart, M.N., Malamud, N., Ellis, W.G. (1972). The Dandy Walker síndrome. A clinic pathological study bases on 28 cases. Neurology, 22, 771-780.

Nazar, N. (1983). Malformación de Dandy Walker .Recuperado el 2 de mayo del 2012 de http://65.182.2.242/RMH/pdf/1982/pdf/Vol50-3-1982-9.pdf

López, H.J.F., García, R.R., Sánchez, V.G. y Pérez, Z.M.A. Hidrocefalia congénita asociada al Síndrome de Dandy Walker. Revisión e informe de un caso. Revista Mexicana de pediatría. Recuperado el 2 de mayo del 2012 de http://new.medigraphic.com/cgi- $\quad$ bin/resumenMain.cgi?IDARTICULO=2722

Osorio, A., Rodríguez, J.G., Pizarro, O., Koller, O., Paredes, A. y Zúñiga, L. Complejo de Dandy Walker, experiencia en el Centro de Referencia Perinatal Oriente. Recuperado el 7 de mayo de 2012 de http://ultrasonografia.cl/us94/04OSORIODW.PDF

Pascual-Castroviejo, I., Velez, A,, Pascual-Pascual, S.I., Roche, M.C. y Villarejo, F. (2002). Dandy-Walker malformation: analysis of 38 cases. Child's Nervous System, 7, 88-97.

Pascual-Castroviejo, I. (1983). Neurología Infantil. Tomo II. Barcelona: Editorial Científico Médica, 1042-1047.

Rodríguez, J. y Cabal, A. (2009). Síndrome de Dandy Walker. Revista de Atención Primaria. Recuperado el 11 de mayo del 2012 de http://www.elsevier.es/sites/default/files/elsevier/pdf/27/27v42n01a13146044pdf $\underline{001 . p d f}$ 